\title{
Knowledge and attitude of postgraduate students in Kenya on ethics in mental health research
}

\author{
B K Amugune, ' B Pharm, M Pharm, PhD, PG Dip; G C Verster, ${ }^{2}$ MB ChB, M Med (Psych), FC (Psych), M Phil (Applied Ethics) \\ ${ }^{1}$ Centre for Medical Ethics and Law, Stellenbosch University, Cape Town, South Africa \\ ${ }^{2}$ Department of Psychiatry, Stellenbosch University, Cape Town, South Africa
}

Corresponding author: B KAmugune (bamugune@yahoo.co.uk)

\begin{abstract}
Background. Most people with mental illness live in developing countries, where a large proportion of these illnesses are undiagnosed and untreated. As effort is made to encourage mental health $(\mathrm{MH})$ research as an avenue to optimise the management of mental illness, this should be accompanied by adequate knowledge, correct attitude and practice on ethical conduct of research. This study reports the knowledge and attitude among postgraduate students in Kenya on ethics in MH research.

Methods. Consenting students undertaking master's degree courses $(n=40)$ with interest in carrying out MH research were assessed using adapted standard tools for assessing knowledge and attitude. Primary comparison is made on the level of knowledge and attitude between the different cohorts.

Results. Participants undertaking postgraduate degrees in medicine, clinical psychology, pharmacy and nursing were individually scored and collectively found to have a medium ( $n=32,79.5 \%)$ or high $(n=8,20.5 \%)$ level of knowledge. The general attitude towards most aspects of the consent process and confidentiality was observed to be appropriate. Low knowledge of international ethics guidelines was observed. Conclusion. Gaps in knowledge and attitude on ethics among the participants have been identified, and this may initiate the process of appropriate interventions necessary in maintenance of ethical practices in the management of mental illness.
\end{abstract}

S Afr J Bioethics Law 2016;9(2):65-68. DOI:10.7196/SAJBL.2016.v9i2.470

Neuropsychiatric disorders, mostly depression, substance abuse and psychoses, comprise about $14 \%$ of the global disease burden. Most people with mental illnesses live in developing countries like Kenya, where a large proportion of those affected remain undiagnosed and untreated ${ }^{[1]}$ This is partly due to a limited number of mental health (MH) professionals and few training opportunities.

Research and research capacity improvement is an avenue that can optimise management of mental illness. However, stigma, discrimination, lack of respect and provision of adequate emotional support as well as human rights violations against those with mental illness are challenges that undermine efforts to address unmet needs. Due to vulnerability exhibited by persons with mental illness, additional or special protection is necessary, especially for those who may have impaired decision-making capacity to give voluntary informed consent. ${ }^{[2]}$

Studies on vulnerable participants require a good understanding and appreciation of the possible ethical dilemmas, and how to appropriately address them to avoid intended or unintended coercion or exploitation. Emerging and validated training techniques should be employed to improve ethical conduct of research, with emphasis on protection of the vulnerable as adequate knowledge and correct attitude is imparted. ${ }^{[3-4]}$

Previous knowledge, attitude and practice studies indicate a large number of students may have negative attitudes towards specialisation in $\mathrm{MH}$ that could easily affect their future ethical handling of research or practice. ${ }^{[5]}$ Each cadre of $\mathrm{MH}$ professionals needs to be sensitised and trained in a targeted manner on research ethics. ${ }^{[6-8]}$ An integrated curriculum approach has been shown to improve on the students' confidence and attitude towards ethics as well as ethical and human rights orientation. ${ }^{[8,9]}$ Medical ethics teaching should, therefore, aim at enriching students with skills on personal view analysis and rational arguments grounded in the philosophy and reasoning behind ethics. ${ }^{[10,11]}$

Few studies on ethics in $\mathrm{MH}$ have been reported in Kenya. A study on mental illness knowledge, attitudes and beliefs of $\mathrm{MH}$ professionals in Kenyan hospitals reported a positive attitude, but with a minimal knowledge base across the different professional groups. ${ }^{[12]}$ With the exception of stigmatisation in depression, ethical issues were not probed in the study. A study on the informed consent process by Kenyan postgraduate students reported that a majority of students used the consent process as their legal protection, as opposed to the participants' protection. ${ }^{[13]}$ This study reports the extent of knowledge and attitude on ethics among Kenyan postgraduate students intending to carry out $\mathrm{MH}$ research.

\section{Methods}

A cross-sectional descriptive study involving consenting postgraduate students with interest in carrying out $\mathrm{MH}$ research as part of their master's research thesis, was carried out. A predesigned and pretested self-administered questionnaire was used to collect information on participants' education and professional background. Knowledge and attitude information on ethics was captured using 
adapted standard knowledge and attitude tools. ${ }^{[8]}$ The knowledge tool consisted of 20 statements, each evaluated by the participant as either true or false. A three-level (agree, disagree or do not know) Likert-type scaling method was employed as the attitude assessment tool on 20 statements on ethics. The participants were to indicate the option that best aligned their view on each statement.

Anonymous but coded data obtained were summarised using descriptive statistics and presented graphically and in the form of tables. Primary comparison was made on the level of knowledge and attitude between the different cohorts of students. Using Pearson's Chi-square test with the score categories as the outcome variable, associations between student factors and other variables were further determined. A pre-determined level of $p<0.05$ was set as the level of statistical significance. Ethics approval for the study was granted by the Stellenbosch University health research ethics committee as well the Kenyatta National Hospital-University of Nairobi ethics review committee.

\section{Results}

\section{Education and professional background}

Out of the 52 postgraduate students who had shown interest in participating in the study, only 40 consented and completed the questionnaires ( $91 \%$ response rate of targeted sample size). The respondents' qualifications included Master of Medicine in Psychiatry (MMed) ( $n=19,46.2 \%)$, Master of Science in Clinical Psychology (MSc Clinical Psychology) ( $n=12,30.8 \%)$, Master of Pharmacy (MPharm) $(n=7,17.9 \%)$ and Master of Science in Mental Health and Psychiatric Nursing (MSc Nursing) ( $n=2,5.1 \%)$.

About $40 \%$ ( $n=16$ ) of the respondents had professional experience of $\leq 5$ years. Seventy percent $(n=28)$ had first heard of ethics in research at undergraduate level, with the rest at postgraduate level. A majority ( $n=34,85 \%)$ had encountered ethics as a class topic and were those who felt undergraduate level was the best time to familiarise with ethics rather than postgraduate or in-service workshops. Nonetheless, only $59 \%(n=24)$ felt confident to ably handle the ethical issues in their $\mathrm{MH}$ research topic.

\section{Knowledge}

For every participant, the total correct response on 20 statements was converted to a \% score. The calculated score was categorised into one of three knowledge levels: low (0 - 50\% score), medium (51 - 74\% score) or high (>75\% score). ${ }^{[14]}$ The scores ranged between $55 \%$ and $90 \%$, with a mean score of $72 \pm 8.6 \%$ (mean \pm standard deviation). Participants were ranked into medium $(n=32,80 \%)$ and high $(n=8,20 \%)$ knowledge categories. The distribution of the participant cohorts into the various knowledge categories is represented in Fig. 1. Half of the high-knowledge participants were MMed students and all MSc Clinical Psychology participants were of medium knowledge. Of the high-knowledge respondents only $37.5 \%$ were confident to handle the ethical issues, in contrast to $65 \%$ of medium scorers.

All participants were aware that a child's refusal to participate in a parent-consented study should be respected, as is the need for review of safety information by research ethics committees before study approval. A large proportion $(n=25,62.5 \%)$ falsely indicated that a child's assent in the absence of parental consent is valid informed consent. Low knowledge in some topic areas was noted, with about $60 \%$ ( $n=24)$ falsely indicating that the main drawback of offering financial incentive to participants is the expense. Similarly, $65 \%(n=26)$ felt consent in studies about the disease condition of mentally incapacitated people could only be facilitated by legally authorised representatives.

With regard to the use of placebo in clinical trials, $47.5 \%(n=19)$ falsely indicated that the control arm should always have a placebo.

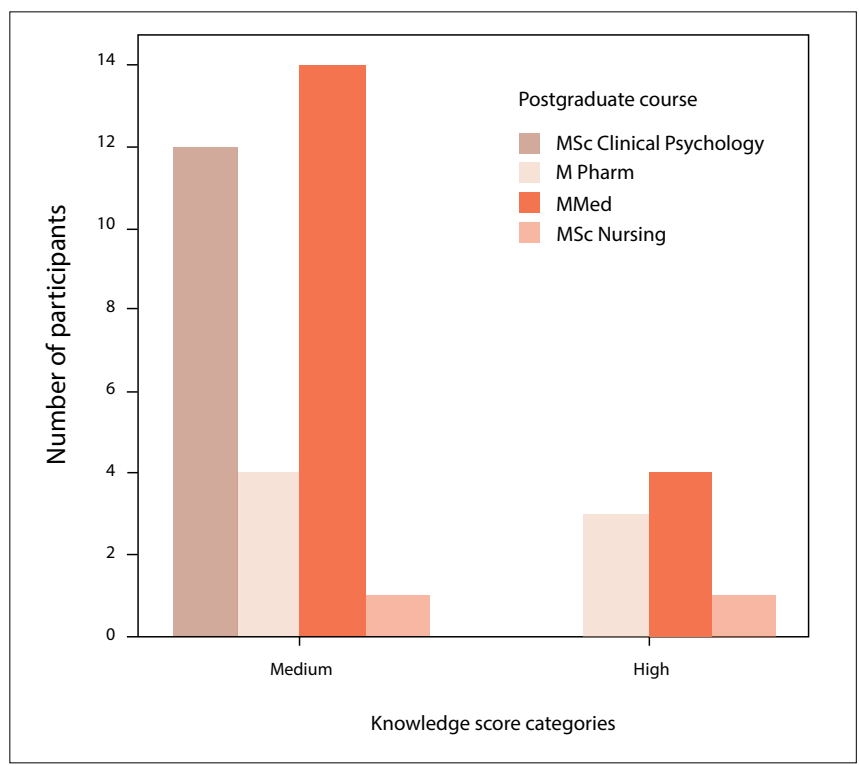

Fig. 1. Distribution of participant cohorts into various knowledge score categories.

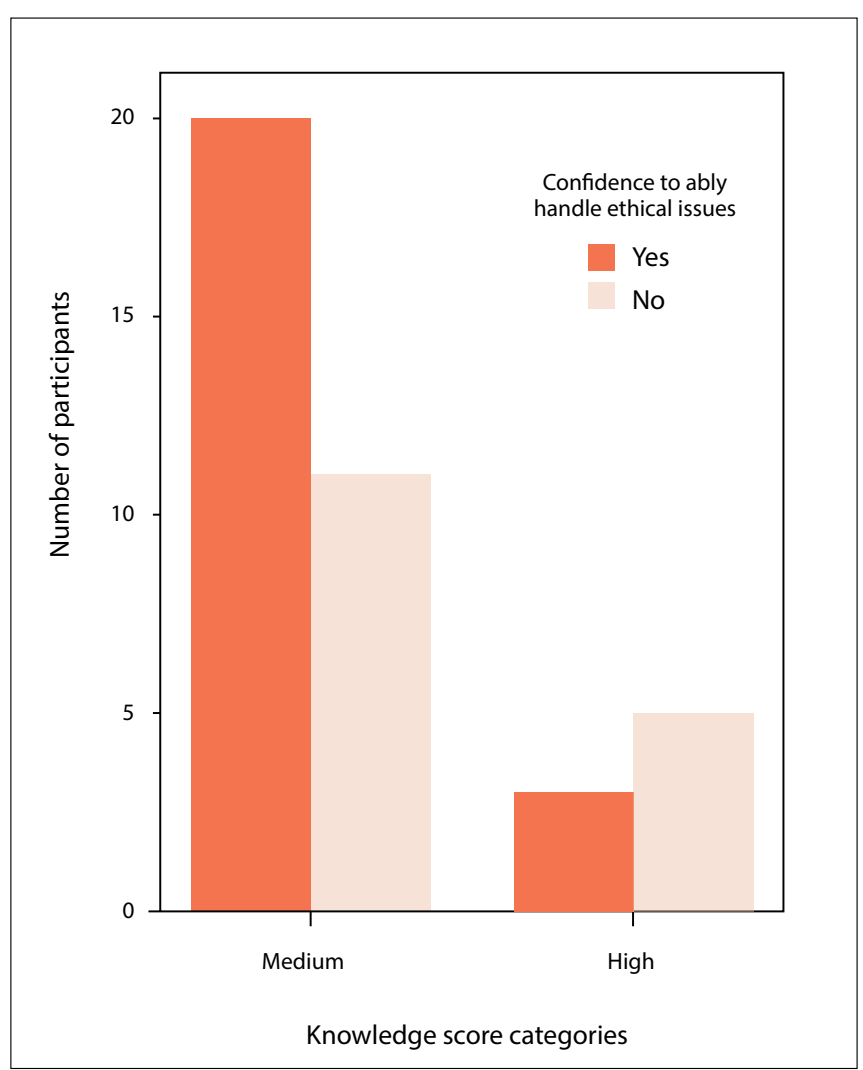

Fig. 2. Confidence to carry out ethical studies by respondents in different knowledge score categories. 


\begin{tabular}{|c|c|c|c|c|}
\hline \multicolumn{2}{|c|}{ Question } & \multirow{2}{*}{$\begin{array}{l}\text { Agree, } \\
n(\%) \\
38(95)\end{array}$} & \multirow{2}{*}{$\begin{array}{l}\text { Disagree, } \\
\boldsymbol{n}(\%) \\
2(5)\end{array}$} & \multirow{2}{*}{$\begin{array}{l}\text { Do not know, } \\
n(\%) \\
0\end{array}$} \\
\hline 1 & $\begin{array}{l}\text { Informed consent form should be a mandatory document in every research } \\
\text { project }\end{array}$ & & & \\
\hline 2 & Informed consent should have accurate information of the research protocol & 39 (97.5) & $1(2.5)$ & 0 \\
\hline 3 & $\begin{array}{l}\text { Persons with mental illness cannot make decisions about their participation in a } \\
\text { research study }\end{array}$ & $25(62.5)$ & $14(35)$ & $1(2.5)$ \\
\hline 4 & $\begin{array}{l}\text { There should be a witness during the informed consent process for persons with } \\
\text { mental illness }\end{array}$ & $33(82.5)$ & $7(17.5)$ & 0 \\
\hline 5 & Informed consent should be over-inclusive & $14(35)$ & $20(50)$ & $6(15)$ \\
\hline 6 & Withdrawal from the study is a right of the participant & $40(100)$ & 0 & 0 \\
\hline 7 & Informed consent is a guard against harm for the participant & $29(72.5)$ & $9(22.5)$ & $2(5)$ \\
\hline 8 & $\begin{array}{l}\text { Informed consent forms should be kept under lock and key and separate from } \\
\text { research records to protect privacy and confidentiality of the participant }\end{array}$ & $34(85)$ & $4(10)$ & $2(5)$ \\
\hline 9 & $\begin{array}{l}\text { Family members should not be asked to force the participant to document the } \\
\text { informed consent }\end{array}$ & $38(95)$ & $1(2.5)$ & $1(2.5)$ \\
\hline 10 & $\begin{array}{l}\text { Confidentially is broken if a doctor/researcher discloses information that places } \\
\text { the patient at risk of injury/harm/illness }\end{array}$ & $29(72.5)$ & $11(27.5)$ & 0 \\
\hline 11 & $\begin{array}{l}\text { A doctor should always ask permission from or inform the patient before he or } \\
\text { she breaks the confidentiality }\end{array}$ & 37 (92.5) & $3(7.5)$ & 0 \\
\hline 12 & $\begin{array}{l}\text { Confidentiality should be maintained whenever possible with the exception of } \\
\text { situations where there is a risk of harm to others }\end{array}$ & $39(97.5)$ & 0 & $1(2.5)$ \\
\hline 13 & $\begin{array}{l}\text { Doctors are a patient's representative and should, therefore, not be expected to } \\
\text { release information about a patient to a third party without a patient's properly } \\
\text { informed consent }\end{array}$ & $37(92.5)$ & $3(7.5)$ & 0 \\
\hline 14 & $\begin{array}{l}\text { Research using individually identifiable health information is important to the } \\
\text { development of medical care }\end{array}$ & $20(50)$ & $19(47.5)$ & $1(2.5)$ \\
\hline 15 & $\begin{array}{l}\text { Researchers should be able to use unidentifiable personal health information } \\
\text { without a person's consent }\end{array}$ & $12(30)$ & $25(62.5)$ & $3(7.5)$ \\
\hline 16 & $\begin{array}{l}\text { Researchers must always get a person's consent to use identifiable health } \\
\text { information }\end{array}$ & $38(95)$ & $2(5)$ & 0 \\
\hline 17 & Confidentiality of health information should be maintained at any cost & $30(75)$ & $10(25)$ & 0 \\
\hline 18 & $\begin{array}{l}\text { Consent to use health information need only be obtained once for all future } \\
\text { research projects }\end{array}$ & $4(10)$ & $35(87.5)$ & $1(2.5)$ \\
\hline 19 & $\begin{array}{l}\text { People should be informed that their health information is being used. They do } \\
\text { not have to give consent }\end{array}$ & $7(17.5)$ & $32(80)$ & $1(2.5)$ \\
\hline 20 & $\begin{array}{l}\text { Advances in genetic understanding of mental illnesses would lead to decreased } \\
\text { discrimination towards these illnesses }\end{array}$ & $35(87.5)$ & $3(7.5)$ & $2(5)$ \\
\hline
\end{tabular}

Further, poor knowledge of international guideline provisions, such as use of active deception (15\%), and the need for aftercare and access (37.5\%) following participation in clinical trials was noted.

Using the knowledge categories as the outcome variable, significant correlation was statistically observed between the participants' primary degree and when they first heard of research ethics $(p=0.018)$, preferred best time to familiarise with ethics and the postgraduate programme enrolled in $(p=0.002)$, as well as confidence to ably handle ethical issues in individual researches and when they first heard of ethics in research $(p=0.049)$. The $\%$ score was found to be positively correlated $(p=0.016)$ to previous encounters of ethics in studies. The relationship between the respondent's knowledge score category and the corresponding confidence to ethically carry out studies in $\mathrm{MH}$ is graphically presented in Fig. 2.

\section{Attitude}

The attitude responses of participants towards ethical issues are summarised in Table 1. All participants agreed on the voluntariness principle as well as the necessity for the informed consent process. Nearly $95 \%(n=38)$ agreed that participants should not be coerced into participation and $72 \%(n=29)$ felt that 'informed consent is a guard against harm for the participant'.

On protection of identifiable health information confidentiality, high concurrence $(95 \%)$ was registered. Over $70 \%(n=28)$ agreed 
that confidentiality is broken if information disclosed places where the patient is at risk of injury or harm. Many respondents rightly felt that informed consent forms should be kept safely away from any direct identifiers. Agreement was obtained in $82.5 \% \quad(n=33)$ of cases on the need for the presence of a witness during the consent process of persons with mental illness. Perception on use of identifiable health information was varied, with agreement on its importance in the development of medical care $(50 \%, n=20)$, use without consent $(30 \%, n=12)$, need for consent before use $(95 \%)$ and maintenance of confidentiality $(72 \%, n=29)$. A large proportion $(87.5 \%, n=35)$ disagreed with the notion that prior primary consent to use health information is sufficient for all future research projects and participants should only be informed without need to re-consent $(80 \%, n=32)$. An approval rating of $87.5 \%(n=35)$ was recorded on the possibility of advances in genetics offering decreased discrimination to those with mental illness.

\section{Discussion}

On average a medium knowledge of ethics is reported for this group of participants, with a score lower than recorded elsewhere using a similar tool. Mishra et al., ${ }^{[8]}$ in an assessment of 32 participants, obtained a score of over $80 \%$ (high knowledge level). Nonetheless, some high individual scores were recorded in key areas of the consent process and confidentiality. Knowledge on participant compensation, confidentiality and consent process in vulnerable groups, such as those with mental illness and children, require attention as well as updates in international ethics guidelines. The major factors that affect knowledge on ethics include the participant's primary degree, the first time they encounter study topics in ethics and their chosen $\mathrm{MH}$ postgraduate degree. The interplay of these factors was shown to affect the confidence of the participant to handle ethical issues in research. An analysis of these factors and their interrelationship may therefore create the intervention opportunity, such as curriculum development or review, in handling of ethical issues. ${ }^{[10]}$

Effort should be made towards addressing the apparent differential in knowledge across the postgraduate sub-groups, as the management of a mentally ill patient requires collective and specific input from each of the professionals represented in this study. Also of concern is the observed low confidence in the handling of ethics among high knowledge scorers in contrast to medium knowledge scorers. The didactic approach of ethics teaching may not be the best and perhaps a skill-based style may be desirable. It is imperative that any knowledge acquisition on ethics should always be accompanied by instilling the requisite attitude and confidence to hopefully inform the eventual practice.

Generally, a desirable attitude towards most aspects of the consent process and confidentiality was observed, with the exception of the handling of identifiable participant health information. Considering that discrimination and stigma are key issues in mental health, the attitude in the handling of health information must be addressed in an effort to protect this vulnerable group. ${ }^{[15]}$

\section{Conclusion and recommendations}

The study participants have exhibited moderate knowledge and appreciably correct attitudes towards ethical issues in key areas of mental health. Areas that may require reinforcing have been identified and may be remedied through curriculum review. The timing of such curriculum or medical training change implementation must come early in the learning cycle. Any interventions must address the link between observed knowledge and attitude to future practice, which was not addressed in the study. This may be addressed through appropriate mentoring by senior colleagues on ethical practice. It is envisaged that lessons learnt from this case study will initiate the process of finding appropriate interventions necessary in achieving and maintaining ethical practices, as the gap in the management of mental illness is bridged.

Acknowledgements. Research reported in this publication was supported by the Fogarty International Centre and Office of AIDS Research, OD and National Institute of Allergy and Infectious Diseases and the National Institute of Mental Health of the National Institutes of Health under Award Number R25 TW 008981 to the ARESA project. The study was carried out, after ethics approval by the Stellenbosch Health Research Ethics Committee (reference S15/05/097 of 17 June 2015), by the Kenyatta National Hospital - University of Nairobi (UON) Ethics and Research Committee (reference P260/05/2015 of 11 June 2015). Gatekeeper permission to involve the postgraduate students as participants was obtained from UON.

\section{References}

1. Ngui EM, Khasakhala L, Ndetei D, Roberts LW. Mental disorders, health inequalities and ethics: A global perspective. Int Rev Psychiatry 2010;22(3):235-244. DOI:10. 3109/09540261.2010.485273

2. Wilen BJ, Appelbaum PS. Subjects' capacity to consent to neurobiological Research. In: Pincus HA, Lieberman JA, Ferris S, eds. Ethics in Psychiatric Research: A Resource Manual for Human Subjects Protection. Washington: American Psychiatric Association, 1998:8-106.

3. Rosenstein DL, Miller FG, Rubinow DR. A curriculum for teaching psychiatric research bioethics. Biol Psychiatry 2001;50(10):802-808.

4. Beresin EV, Baldessarini RJ, Alpert J, Rosenbaum J. Teaching ethics of psychopharmacology research in psychiatric residency training programs. Psychopharmacology 2003;171(1):105-111. DOI:10.1007/s00213-003-1434-x

5. Voinescu B, Szentagotai A, Coogan A. Attitudes towards psychiatry: A survey of Romanian medical residents. Acad Psychiatry 2010;34(1):75-78. DOI:10.1176/ appi.ap.34.1.75

6. Hariharan S, Jonnalagadda RR, Walrond E, Moseley H. Knowledge, attitudes and practice of healthcare ethics and law among doctors and nurses in Barbados. BMC Med Ethics 2006;9(7):E7. DOI:10.1186/1472-6939-7-7

7. Hicks LK, Lin Y, Robertson DW, Robinson DL, Woodrow SI. Understanding the clinical dilemmas that shape medical students' ethical development: Questionnaire survey and focus group study. BMJ 2001;322(7288):709-710. DOI:10.1136/bmj.322.7288.709

8. Mishra NN, Bhatia T, Kumar N, et al. Knowledge and attitudes of mental health professionals regarding psychiatric research. Indian J Med Res 2014;139(2):246251.

9. Self D, Baldwin D, Wolinsky F. Evaluation of teaching medical ethics by an assessment of moral reasoning. Med Educ 1992;26(3):178-184. DOI:10.1111/j.1365-2923.1992.tb00151.x

10. London L, McCarthy G. Teaching medical students on the ethical dimensions of human rights: Meeting the challenge in South Africa. J Med Ethics 1998;24(4):257262. DOI:10.1136/jme.24.4.257.

11. Ndetei DM, Khasakhala L, Mutiso V, Mbwayo AW. Knowledge, attitude and Practice (KAP) of mental illness among staff in general medical facilities in Kenya: Practice and policy implications. Afr J Psychiatry 2011;14(3):225-235. DOI:10.4314/ajpsy.v14i3.6

12. Wagoro MCA, Bhatt KM. An audit of the informed consent process in postgraduate dissertation studies at the College of Health Sciences, University of Nairobi, Kenya. S Afr J Bioethics Law 2012;5(1):45-50.

13. Kumar R, Mehta S, Kaira R. Knowledge of staff nurses regarding legal and ethical responsibilities in the field of Psychiatric nursing. Nurs Midwif Res $J$ 2011;7(1):1-11.

14. Ligüy $M$, Ligüy $D$, Oktay I. Ethical decision making in dental education: A preliminary study. BMC Med Ethics 2015;16(7):52. DOI:10.1186/s12910-0150046-4

15. Rogers W, Braunack-Mayer A. Handling information. Some ethical issues. Aust Fam Physician 2000; 29(8):806-808. 